\title{
Preparations for the remeasurement of the Eötvös- experiment
}

\author{
Lajos Völgyesi ${ }^{* 1}$ György Szondy ${ }^{2}$, Gyula Tóth ${ }^{1}$, Gábor Péter ${ }^{3}$, Bálint Kiss ${ }^{3}$, \\ Gergely Barnaföldi ${ }^{4}$, László Deák ${ }^{4}$, Csaba Égetö ${ }^{1}$, Edit Fenyvesi ${ }^{4}$, Gyula Gróf ${ }^{5}$, \\ László Somlai ${ }^{4}$, Péter Harangozó ${ }^{3}$, Péter Lévai ${ }^{4}$, Péter Ván ${ }^{4,5}$ \\ ${ }^{1}$ Budapest University of Technology and Economics (BME), Dept. of Geodesy and Surveying, Hungary \\ ${ }^{2}$ Independent researcher, Society for the Unity of Science and Technology, Hungary \\ ${ }^{3}$ BME, Dept. of Control Engineering and Information Technology, Hungary \\ ${ }^{4}$ Wigner Researche Centre for Physics, Hungary \\ ${ }^{5}$ BME, Faculty of Mechanical Engineering, Dept. of Energy Engineering, Hungary \\ E-mail: volgyesiaeik. bme.hu
}

This work was supported in part by the National Research, Development and Innovation Office - NKFIH 124286 and 124366. (Hungary).

\begin{abstract}
Between 1906 and 1908 Roland (Loránd) Eötvös and his colleagues Dezső Pekár and Jenő Fekete made measurements with revolutionary precision (the EPF measurement) for validating the equivalence of gravitational and inertial mass. Almost 80 years later, in 1986, Ephraim Fischbach and his colleagues reanalyzed the results of the EPF measurement and discovered a correlation between the small violations and some atomic parameter. Finally they were unable to reproduce this correlation experimentally, but there is still no valid explanation of these differences in the EPF results. Our analysis of the EPF experiment pointed to a possible bias that justifies repeating the tests under better conditions and using modern new technology. Planning and preliminary measurements started at July of 2017. Participants are from Wigner Research Centre for Physics of the Hungarian Academy of Sciences, Department of Geodesy and Surveying of Budapest University of Technology and Economics (BME), Society for the Unity of Science and Technology (SUST), and the Department of Control Engineering and Information Technology of BME in cooperation with other organizations, departments and experts. After more than one year of thoughtful preparation, in December 2018 preliminary tests have been started in a controlled and undisturbed environment of the Jánossy Underground Physics Laboratory at KFKI, 30 meters below ground level. This paper gives a brief overview of the basic physics, history, preparations and present status of the new experiments. Another good reason for repeating the EPF measurements is that 2019 - as the 100th anniversary of Eötvös's death - is referred as "Eötvös year".
\end{abstract}

International Conference on Precision Physics and Fundamental Physical Constants - FFK2019 9-14 June, 2019

Tihany, Hungary

*Speaker

(c) Copyright owned by the author(s) under the terms of the Creative Commons Attribution-NonCommercial-NoDerivatives 4.0 International License (CC BY-NC-ND 4.0). 


\section{Introduction}

Lorand Eötvös and his colleagues Dezső Pekár and Jenő Fekete executed a series of measurements (EPF experiment) from 1906 till 1908 to validate the equivalence of gravitational and inertial mass [1]. Almost 80 years later, in 1986, Fischbach et al reanalyzed their data and found a systematic violation that was only a couple of times larger than the uncertainty of the measurement. This violation has not been reproduced or explained by any later, more accurate, but partially different measurement methods.

Via our new analysis of the EPF data, we have found a possible source of systematic error $[2,16]$ that justifies repeating the experiment under better conditions and using current technology. The measurements are executed in the Jánossy Underground Physics Laboratory (JUPL) at Wigner Institute Budapest, at a depth of $30 \mathrm{~m}$, under suitable, controlled conditions. The Eötvös Year (the 100th anniversary of Lorand Eötvös's death) ensures special attention to the new measurements. In our current study, we briefly review the history and report the preparation of the measurements.

\section{History}

The main concept of the EPF measurement was that the weight force is the sum of a gravitational force toward the center of the Earth and the centrifugal force perpendicular to its axis of rotation. If gravity depends on the composition of the test body, then the direction of the resulting weight force will also depend on it. This small difference can be detected via an East-West orientated torsion balance (TB). The measurements they made showed only negligible differences, which appear to be a few times the uncertainty of the measurement $\left(10^{-9}\right)$.

Almost 80 years later, in 1986, Fischbach et al. found that these small differences were not completely random but could be written as a linear function of the binding energy of the nuclei and suggested the existence of a short-range force $[3,4,5]$. The assumption has induced serious debate. The results of Eötvös were analyzed in detail and new, more accurate measurements were made e.g. by the Eöt-Wash group at University of Washington [9], Cowsik and his team at the Tata Institute of Fundamental Research [20] and others, but they did not reveal the predicted Yukawa-like, finite-range new force.

However, for long distances no one doubted the equivalence of gravitational and inertial mass, since more precise measurements have been made since Eötvös. Prior to Fischbach, Robert Dicke et al. confirmed the equivalence principle to the accuracy of $(1.3 \pm 1) \times 10^{-11}$, and Braginsky et al. to $(0.3 \pm 0.9) \times 10^{-12}[6,7,8]$.

Robert Dicke et al. introduced several innovations. First, a north-south pendulum measured the material dependence of the relationship between the attraction of the Sun 
and the centrifugal force of the Earth's orbit. The advantage of this is that - due to the 24-hour periodicity of the signal - the TB did not have to be rotated during the measurement. Also a triangular balance was used which was more insensitive to gradient effects, and the balance - consisting of a gold-aluminum materials - was placed in a vacuum, and was damped and balanced by means of two electrodes via a feedback loop. The continuous drift of the quartz fiber was also compensated electrostatically. Data recorded during several days of measurement cycles, and has been processed by a computer to filter out disturbance signals caused by temperature fluctuations and mechanical vibrations (A building construction was about 30 meters from the measuring site causing the data between 8:30 and 16:30 on working days un usable).

Braginsky and his associates used a similar method, but the sensitivity of the instrument was significantly enhanced by increasing the length of the tungsten fiber extremely to about $3 \mathrm{~m}$.

After 1986, the rise of the Fifth Force gave new impulse to these experiments. Since 1990, the Eöt-Wash Group (University of Washington) has carried out similar, increasingly accurate measurements. In their latest and most accurate pendulum measurements, they achieved accuracy is $10^{-13}[9,19]$. A special feature of the measurements is that they measure extreme gradients to test Yukawa interaction up to 1 meter range. The gradient effects that occur are carefully compensated for. The group also carried out measurements where the Sun and the Milky Way were considered to be the attractor [19].

By measuring the Earth-Moon distance the Lunar Laser Ranging (LLR) Experiment compares the gravitational acceleration of Earth and Moon to the Sun. Measurement shows that the acceleration of the two bodies is identical within the uncertainty of $10^{-13}$. The result may have more implications. If the ratio of inertial and gravitational mass changes due to the gravitational self-energy it would mean the violation of strong equivalence principle. But this kind of gravitational "binding energy" far less than the binding energy in the nuclei, therefore the LLR experiment validates strong equivalence principle within the uncertainty of $(2.3 \pm 3.2) \times 10^{-4}$. In relation to this measurement one can refer to the assumption that the composition of the Earth and the Moon is different from each other because the Earth might have a iron-nickel core similar to the material of iron meteorite, while the moon has no such core [9]. In this case the weak equivalence principle could be validated with higher accuracy. However, the mentioned geochemical meteorite model is not consistent with today's geophysical knowledge as it can't explain for example the vanishing of shear stresses in the outer core. The so-called astrophysical model is more realistic which states that there is no significant difference in the material composition of the Earth's crust and the core, but the difference is the phase transition caused by the high pressure [10]. Due to the smaller mass of the Moon there is no such high pressure that could cause an Earth-like 
phase transition, consequently Moon has no such core and magnetic field as the Earth. Observing the rocks from the Moon there is no significant difference of the composition and age of the two planets. It supports the theory that both planets were created at the same time from the same source of material, which implies that their composition is also the same [11]. Thus, the LLR the experiment is unlikely to be suitable for validating the weak equivalence principle.

The MICROSCOPE experiment, which has been collecting data since December 2016, plans to improve the accuracy of the experiments by two orders of magnitude, utilizing the difference in relative acceleration of test masses orbiting the Earth on slightly different orbits. The planned "Galileo Galilei" experiment would, in tandem with a zero-gravity torsion pendulum in space, improves the order by two orders of magnitude and verify the validity of the weak equivalence principle to $10^{-17}$ [12].

Because of these high precision tests EPF measurements were not repeated in their original form and with original equipment, and after more recent measurements using more sophisticated methods, the reason for the systematic discrepancy observed in Eötvös's work [6] was ignored. Therefore the violation of EPF in these experiments is not completely excluded [17].

We assume that the systematic error in the Eötvös measurements is due to the higher order effect of the gravity gradient, which results in a force dependent on the shape of the applied samples. The aim of our new EPF experiment is not only to confirm the assumption of systematic error mentioned above, but - taking this shapeeffect into account- adds new aspects to the current most accurate ground measurements as well [18].

\section{The principle of the EPF experiment}

Earth's gravity vector $\boldsymbol{g}$ has two main components, the gravitational force $\mathbf{F}_{\mathrm{T}}=m \mathbf{G}$ and the rotational centrifugal force $\mathbf{F}_{\mathrm{C}}=m \mathbf{C}$, where $\mathbf{G}$ is the gravitational and $\mathbf{C}$ is the rotational field intensity. The basic assumption of Eötvös was that the centrifugal force of $\mathbf{F}_{\mathrm{C}}$ is independent of material composition, but that the gravitational force of $\mathbf{F}_{\mathrm{T}}$ might depend on it. Consider the placement of various materials (e.g. gold and aluminum) at the surface of the Earth, as shown in Fig. 1. The two different bodies have exactly the same mass $\left(m=m^{\prime}\right)$. It is assumed that both bodies are subject to the same centrifugal force $\mathbf{F}_{\mathrm{C}}$, but the Earth exerts gravitational force $\mathbf{F}_{\mathrm{T}}$ on mass $m$ and $\mathbf{F}_{\mathrm{T}}^{\prime}$ on mass $m$ '. Accordingly, as shown in Fig. 1, the gravity force on the mass $m$ is $\mathbf{g}$ and the gravity force on the mass $m^{\prime}$ is $\mathbf{g}^{\prime}$.

The idea of the EPF experiment [1] was to compare the horizontal component of gravitational force $m \mathbf{g}$ due to the Earth acting on different materials or samples with the horizontal component of centrifugal force $m \mathbf{C}$. Centrifugal force is assumed composition independent hence if gravitational force depends on material composition, the imbalance of horizontal forces can be detected with a TB. 


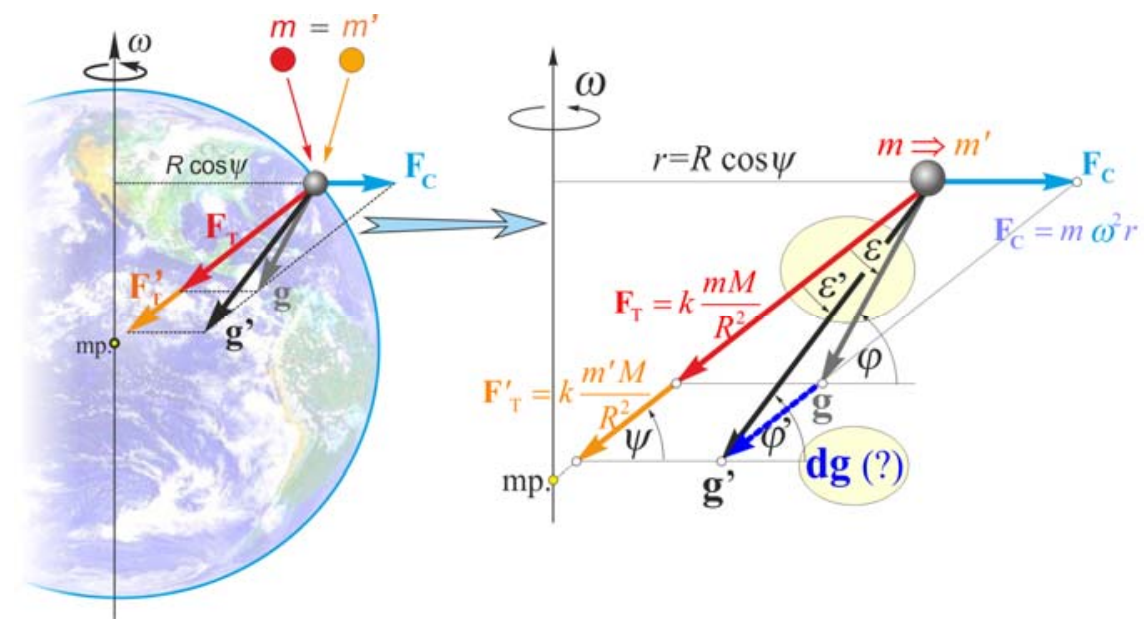

Fig. 1. Assumed difference of gravity in case of different materials

The $\mathbf{F}_{\mathrm{C}}$ centrifugal force acting on the bodies rotating together with the Earth is perpendicular to the axis of rotation, and on the Northern hemisphere the horizontal component of $\mathbf{F}_{\mathrm{C}} \sin \varphi$ facing south, as shown in Fig. 2.

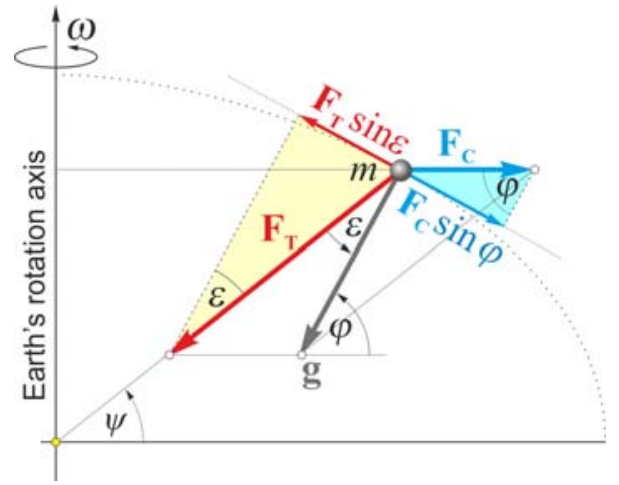

Fig. 2. Horizontal components of forces

The size of the $\mathbf{F}_{\mathrm{C}} \sin \varphi$ component depends on the geographic latitude $\varphi$ of the measurement site. This force is in balance with the northward force $\mathbf{F}_{\mathrm{T}} \sin \varepsilon$, which is the horizontal projection of the gravitational force $\mathbf{F}_{\mathrm{T}}$ acting on the body. The angle $\varepsilon$ in Fig. 2 is the angle enclosed by the gravity force $\mathbf{g}$ and the gravitational force $\mathbf{F}_{\mathrm{T}}$. Assuming that the gravitational force of $\mathbf{F}_{\mathrm{T}}$ may depend on material composition, Eötvös introduced the notation of material quality factor:

$$
\eta=2 \frac{\left(m_{s} / m_{t}\right)-\left(m_{s}^{\prime} / m_{t}^{\prime}\right)}{\left(m_{s} / m_{t}\right)+\left(m_{s}^{\prime} / m_{t}^{\prime}\right)}
$$

Thus, if we take $\eta=0$ for some reference material (eg for water), the force of gravitation on the another body will change according to the relation $(1+\eta) \mathbf{F}_{\mathrm{T}}$.

If $\eta \neq 0$, the balance of two forces shown in Fig. 2 is lost due to the difference of gravitational and inertial masses, so the mass $m$ ' will be affected by the force $\eta \mathbf{F}_{\mathrm{T}} \sin \varepsilon$ pointing to northward, which will result in a small turning of the balance beams. Thus, 
the main question of the Eötvös experiment is whether it is observable a small turning of the balance beams due to the supposed different forces acting on the upper and lower masses inside the torsion balance if we replace the lower mass with another material.

A possible measurement strategy of the EPF experiments can be seen in Fig. 3.

In the first step we set up the TB in the E-W direction $\left(\alpha=90^{\circ}\right.$ azimuth), and then after the damping time the position of the balance bar can be read.

In the second step the TB must be rotated by 180 degrees $\left(\alpha=270^{\circ}\right)$, and then after the damping time the position of the balance bar can be read again.

In the third step the TB must be rotated back to $\alpha=90^{\circ}$ and the suspended lower gold mass should be replaced by a different material but the same weight, and then waiting for the damping time the position of the balance bar can be read.

Finally, in step 4, with the replaced mass the TB must be rotated by 180 degrees $\left(\alpha=270^{\circ}\right)$, and then after the damping time the position of the balance bar can be read again.

In practice, we measure with a double balance - which consists of two single balances bound together in anti-parallel position - and a more complicated measurement strategy, where we also exchange the masses between the two balances [15].

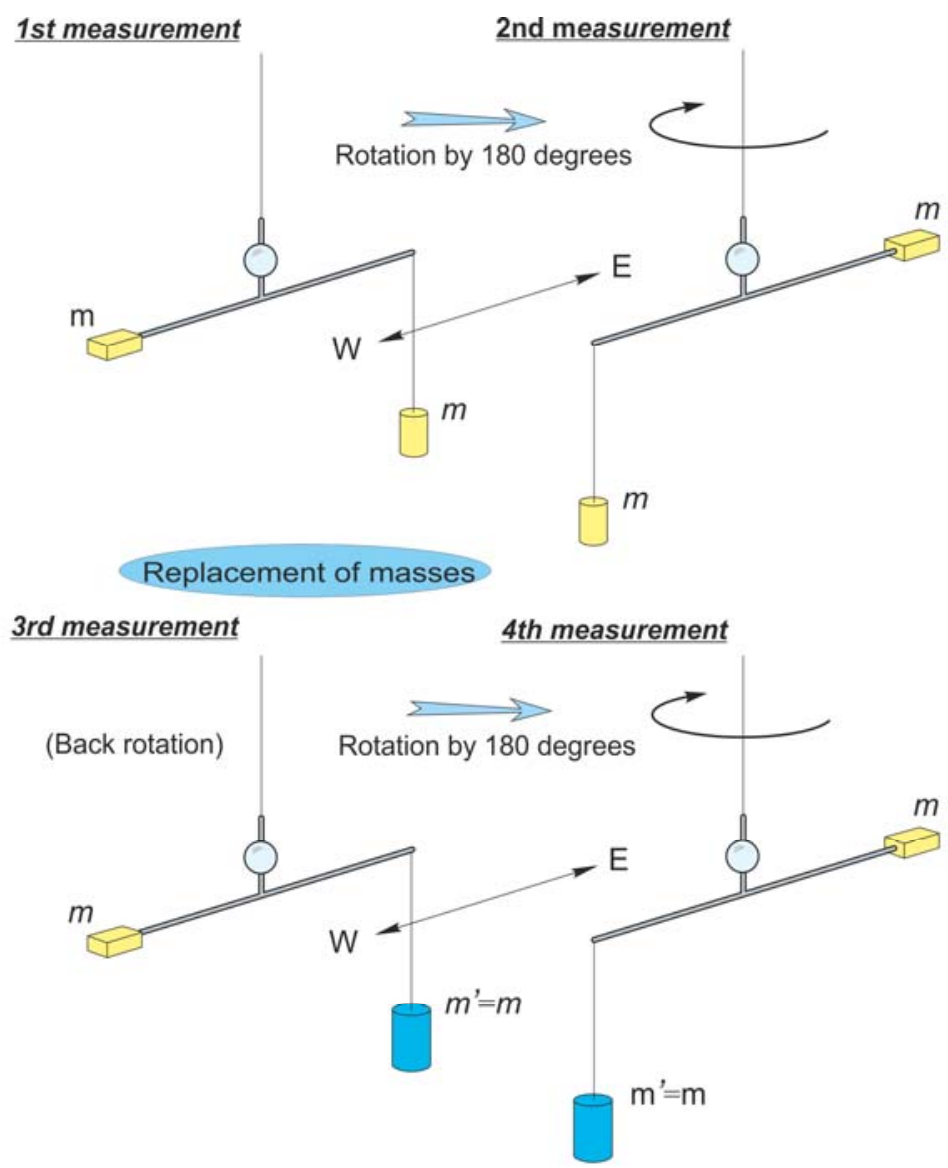

Fig. 3. Strategy of EPF measurement by exchanging masses 


\section{Preparations for the new EPF measurements}

Our goal was to repeat the EPF experiment in better circumstances, with at least one order higher precision using the original method of Eötvös, having very limited budget. There were three different instruments shown in Fig. 4 which appeared to be suitable for performing our measurements. State of the art instrumentation of these instruments offered the requested enhancement of precision on a very low cost level.
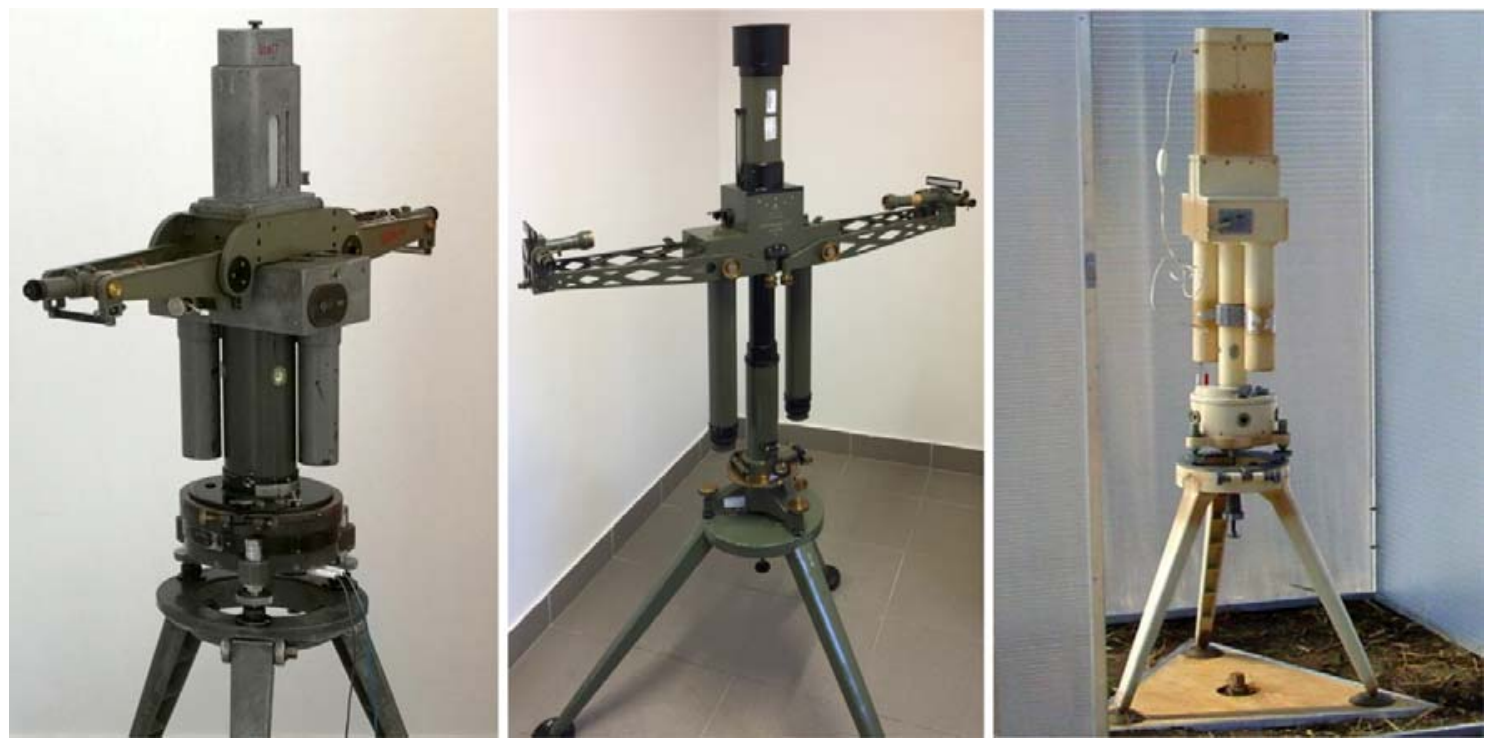

Fig. 4. Three types of historical torsion balances suitable for the EPF measurements. On the left side is the AutERBal, in the middle the Eötvös-Pekár and on the right side the E54 TB can be seen.

On the left side of Fig. 4. the AutERBal (Automatic Eötvös-Rybar Balance) can be seen, which had a $40 \mathrm{~min}$ recording period. The automatic rotation of the instrument to different azimuths was done by a clock mechanism and data reading was photographically recorded. The automation made the constant presence of the observer unnecessary, but the complex control structure needed constant attention [13].

The other type is the Eötvös-Pekár torsion balance which was designated as the Small Original Eötvös G-2, can be seen in the middle part of the Fig. 4. This instrument had three variants; they differed from each other in the length of the torsion wires only. Our model which was manufactured in 1930 has $30 \mathrm{~cm}$ long torsion wire. The developer of this instrument, D. Pekár, with long experiences in field measurements, strived to achieve high accuracy and simplicity of the instrument, and therefore preferred manual rotation and visual reading rather than automatic operation. The only disadvantage of the Pekár balance is the need for the constant presence of the observer [13]. 
The third type of TB available to our measurements can be seen on the right side of Fig. 4. This instrument designated E54 was designed for geophysical prospecting in the 1950s.

We compared the stability (drift) and sensitivity as well as the possible instrumentation (automatic rotation and read-out) of the three balances. Based on our tests, among them we found the Eötvös-Pekár torsion balance to be the most suitable instrument for the remeasurement of the Eötvös experiment. This instrument has the highest sensitivity and reliability, moreover it has the most easily replaceable masses. Thus, our further developments were primarily focused on this instrument.

Since this Eötvös-Pekár balance has not been used for a long time, the first important step was to renovate, repair, check the usability, then to adjust and calibrate of the instrument [14].

In order to minimize the drift of the torsion wires, they were continuously loaded for nearly a year besides the unlocked position of the balance beams. With the long-term tensile loading of the wires, both of them were able to achieve a nearly drift-free state.

The most important requirement for successful measurements is to eliminate the environmental effects as far as possible. The most dangerous sources of error for EPF measurements are the mass of the observer, ground vibrations, temperature changes, and gravity gradients around the instrument. The latter problem can be remedied by selecting an appropriate site for the experiments and measuring the Eötvös tensor elements containing the second derivatives of gravity potential in the environment next to the instrument. By choosing the right location, the stability of the ambient temperature can be achieved and the ground vibrations caused by traffic can partly be eliminated too [18].

The site of our experiment is the Jánossy Underground Physics Laboratory of Wigner RCP, suited $30 \mathrm{~m}$ depth below the ground surface. The temperature stability of this site is satisfactory, the daily change is only a few hundredths of degrees, and the amplitude of the microseismic ground vibrations is also sufficiently small. Environmental factors were continuously recorded with several temperature, pressure, humidity, infrasound sensors, including a Güralp 3T three-axis broadband seismograph.

The biggest enemy of the high precision TB measurements with original methodology is the man himself. The mass of the observer's body changes the damped position of the TB, and going toward the instrument the noise of the observer's steps cause ground vibrations, which also disturbs the damped position of the TB. Solutions for these problems can be achieved with two important enhancements: by applying computer-controlled scan on a CCD sensor instead of visual reading and by using remote-controlled rotation mechanics to rotate the TB into various azimuths.

By mounting a CCD sensor instead of visual reading (see Fig. 5.), we can determine the very precise position of the balance arm by evaluating the resulting digital image. The original optical results in a readout of the equilibrium position with accuracy 
of 0.1 scale division, but now with CCD sensors and continuous reading (up to 10 readings $/ \mathrm{sec}$ ) with accuracy of 0.002 scale division is possible. Scale division is exactly $1 / 3 \mathrm{~mm}$, while the scale-mirror distance is $450 \mathrm{~mm}$. From these numbers 0.002 scale division means $0.3^{\prime \prime}$. To improve the reading accuracy, we have designed a new barcode scale with a computer evaluation process.

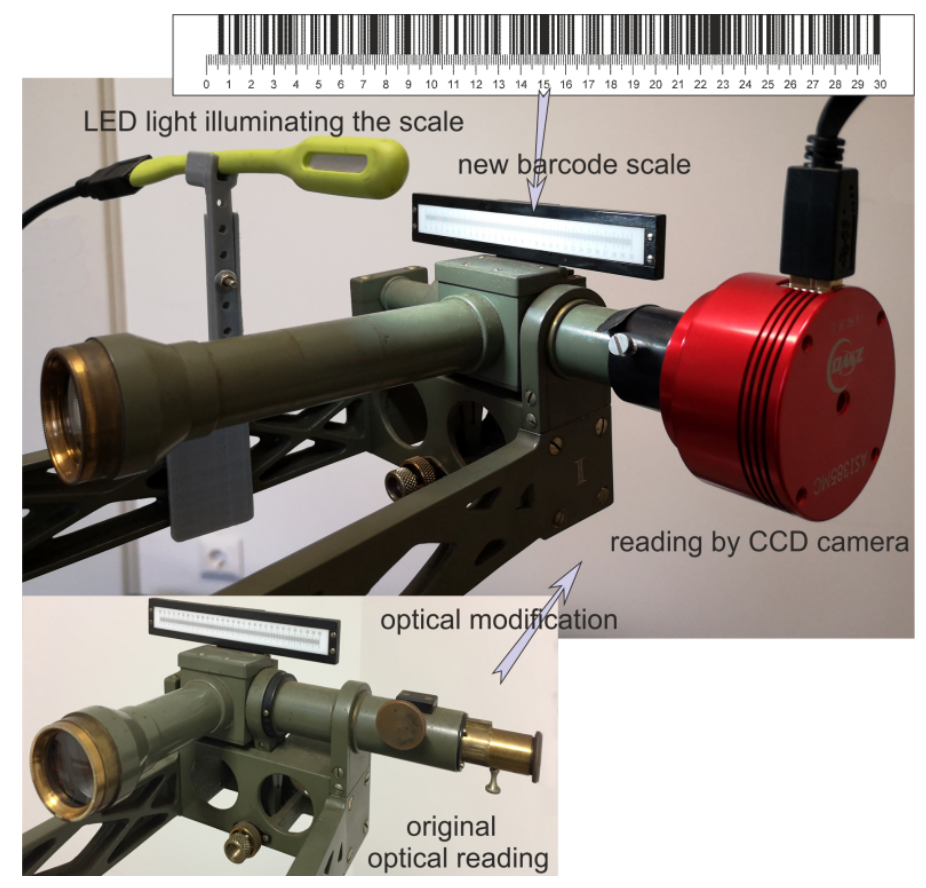

Fig. 5. Reading the scale of the torsion balance by CCD camera

An essential requirement for the experiment is the precise determination of the damped position of the balance arm. In the original EPF experiment this equilibrium position was determined after a waiting time of 40 to 60 minutes by a single visual reading. Unlike the original experiment, the equilibrium position is determined now by fitting a theoretical damping curve to the real damping curves of the TB arms. Therefore the damped position can be determined with a higher accuracy on the basis of more data, and on the other hand, by continuously monitoring of the oscillation of balance bars, we can get important information about the behavior of the TB during measurements.

The most critical element of mechatronic solutions is the construction of a unit for remote rotation of the instrument. For rotation of the TB into various measuring azimuths, the structure shown in Fig. 6 was made, applying a special motor, which is connecting to the upper part of the free rotating TB via a ribbed belt drive.

The rotating mechanism has low weight and low metallic content. We used 3D printing technology (using PLA filament) to produce most of the parts. A low power DC motor rotates the TB through a timing belt with a 1:10 gear ratio hence its magnetic field has negligible influence. A cascade position control loop is realized on the 
microcontroller with a $100 \mathrm{~Hz}$ sampling frequency which meets the desired closed-loop requirements. A scripting language has been also designed allowing the definition measurement protocols for several days during which the pendulum is subsequentially and/or repeatedly rotated to different azimuths.

Since the precise reading of the azimuth value is an important requirement for the overall measurement accuracy, a high resolution absolute optical encoder is used with an accuracy under 3" (arcsec). The rotating mechanism includes a timing belt transmission therefore the azimuth measurements happen directly on the vertical rotating axis of the instrument, in order to avoid the influence of the belt flexibility on accuracy. Let us note that the precision requirement for the closed-loop angular positioning is far less stringent compared to the azimuth measurement precision since the specified repetition accuracy is $\pm 2^{\circ}$ ( $\mathrm{deg}$ ).

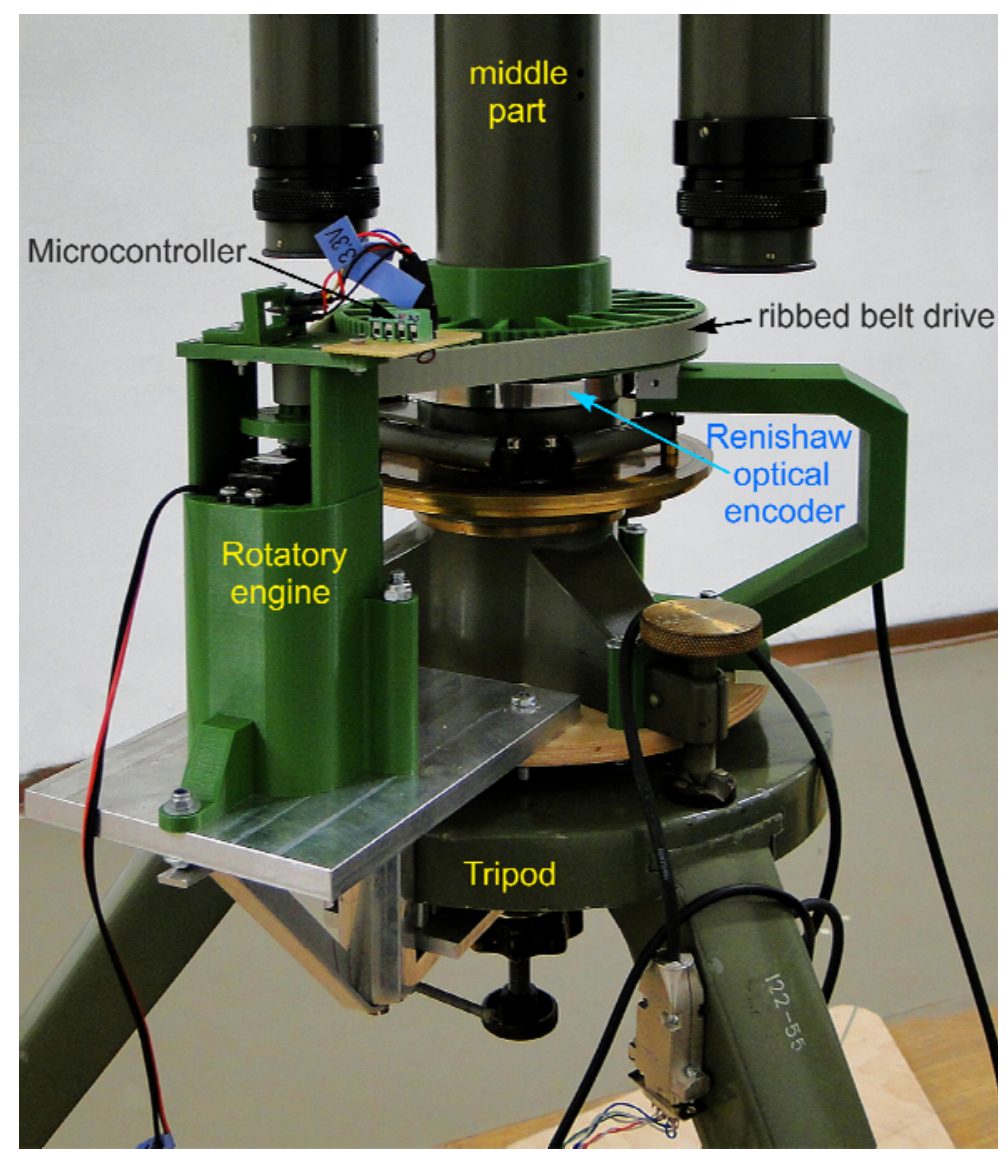

Fig. 6. The rotation mechanism of the torsion balance

The architecture of the data acquisition and control system (DACS) is depicted in Fig. 7.

The task of DACS is to $\log$ the information gathered by the sensors (including the cameras) and to periodically update the duty cycle of the PWM signal sent to the motor allowing the automatic azimuth change of the TB. The real-time data acquisition of the 
sensors (excluding the cameras) and the calculation of the feedback law are realized by a microcontroller based embedded device. The cameras feed the images directly to a PC where they are saved and then processed. The image processing is executed real-time. All data logged during the experiments are stored on a dedicated server for possible later analysis.

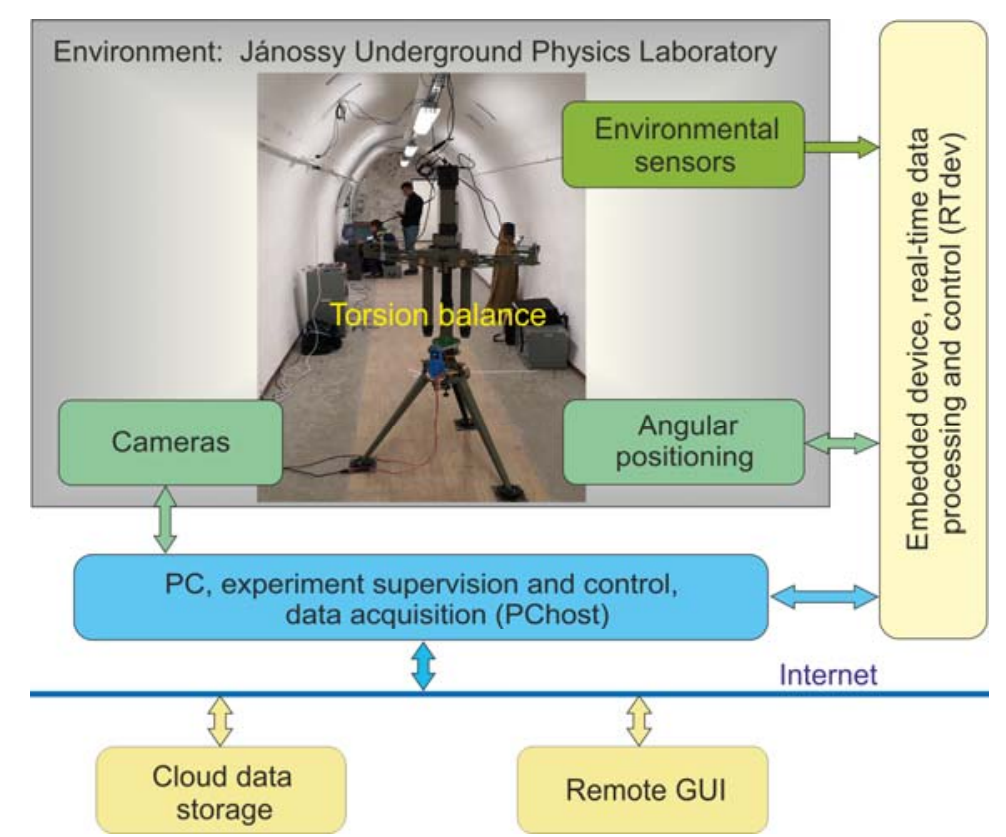

Fig. 7. The DAC system built around the torsion balance

\section{Summary}

In the EPF experiment Roland Eötvös and his coworkers determined the error of the equivalence of inertial and gravitational masses so that it was larger than the errors of the individual measurements. In 1986 E. Fischbach and his coworkers explored a systematic deviation in the data. The origin of this deviation is still mysterious $[6,16$, 17]. According to our hypothesis, gravity gradient effects could be a reason, becasue the original samples of Eötvös were cylinders with uniform diameter and higher order gravity gradient originated force depends on the shape of the samples. This possible systematic error in the EPF measurements can be verified repeating the experiment with better conditions and modernized technology.

Our improved small original Pekár type Eötvös balance started the weak equivalence principle tests in summer 2019 in the Jánossy Underground Physics Laboratory (JUPL), -30 meters underground. An optical CCD readout system with built in scale illumination and a remote rotation control was installed, perfected and most of the related control, readout and evaluation software has been written and tested. Also the reconstruction of the underground laboratory facility is finished. The prepared Eötvös-Pekár balance started the calibration measurement in JUPL in 20 December 
2018 and the fine tuning of the software was accomplished. In spring 2018 a Renishaw code ring was installed to the automatic rotation mechanism in order to improve precision of the horisontal angle determination. We have also elaborated a method to exchange the test masses. As we have reported above, the equivalence principle test has been started with gradient corrected aluminum and copper test masses [16]. The testing method applies the Sun centered fixed arrangement, without rotation.

Here at the conference we have reported the results of the first two weeks. Up to now we did not detect any deviation from the equivalence principle.

The aim of our continued experiments is the improve the sensitivity of the EPF measurements at least by one magnitude. Then, with the help, of both normal and gradient corrected test masses and known gravitational environment one can decide whether the observed systematic error in the EPF measurements was due to gradient effects or some other mechanisms could play a role. The possibility of such mechanisms cannot be excluded, because none of the other TB equivalence tests applied the original device and the original technology of Eötvös and his coworkers as it was emphasised several times by Fischbach.

\section{References}

[1] R. Eötvös, D. Pekár, E. Fekete, Beiträge zum Gesetze der Proportionalität von Trägheit und Gravität. Annalen der Physik 373, (1922) 11-66.

[2] G Tóth, Explanation of the EPF experiment in terms of gravity gradients. arXiv (2018) https://arxiv.org/abs/1803.04720.

[3] E. Fischbach, D. Sudarsky, A. Szafe, C. Talmadge, S. H. Aronson, Reanalysis of the Eötvös experiment, Physical Review Letters, 56/11, (1986), 3.

[4] L. Bod, E. Fischbach, G. Marx, M. Nárayné Ziegler, One hundred years of the Eötvös experiment, Fizikai Szemle, 42/3 (1992), 94-101. (In Hungarian)

[5] P. Király, The Eötvös-Pekár-Fekete experiments and their current influence, Fizikai Szemle, 57/1 (2007), 1. (In Hungarian)

[6] A. Franklin, E. Fischbach, The rise and fall of the fifth force: Discovery, pursuit, and justification in modern physics, (2016), Springer.

[7] P. G. Roll, R. Krotkov, R. H. Dicke, The equivalence of inertial and passive gravitational mass. Ann. Phys. 26 (1964) 442.

[8] V. B. Braginsky, V. I. Panov, Verification of Equivalence Principle of inertial and Gravitational Mass. Zb, Eksp. Theor Fiz. 61 (1971) 873.

[9] E. G. Adelberger, J. H. Gundlach, B. R. Heckel, S. Hoedl, S. Schlamminger, Torsion Balance Experiments: A Low-energy Frontier of Particle Physics. Progress in Particle and Nuclear Physics, 62 (2009), 102-134.

[10] L. Völgyesi, M. Moser M, The Inner Structure of the Earth. Periodica Polytechnica Chem. Eng., 26 (1982), 155-204.

[11] L. Völgyesi, M. Moser, The Inner Structure of the Moon. Periodica Polytechnica Chem.Eng., 29 (1985) 59-70.

[12] A. M. Nobili, A. Anselmi, Relevance of the weak equivalence principle and experiments to test it: Lessons from the past and improvements expected in space, Physics Letters A, 382 (2018), 2205-18.

[13] Z. Szabó, The history of the 125 year old Eötvös torsion balance. Acta Geodaetica et Geophysica 51, (2016) 273-293.

[14] L. Völgyesi, C. Égető, S. Laky, G. Tóth, Z. Ultmann, Reconstruction of a torsion balance, and test measurements in the Mátyás cave in Budapest. Geomatikai Közlemények, XII (2009),71-82. (In Hungarian) 
[15] L.Völgyesi, G. Szondy G. Tóth, G. Péter, B. Kiss, L. Deák, C. Égetõ, E. Fenyvesi, G. Gróf, P. Ván P, Preparations for the remeasurement of the Eötvös-experiment. Magyar Geofizika 59/4, (2018), 165-179. (In Hungarian)

[16] G. Tóth, L. Völgyesi, G. Szondy, G. Péter, B. Kiss, G. Barnaföldi, L. Deák, C. Égető, E. Fenyvesi, G. Gróf, L. Somlai, P. Harangozó, P. Lévai, P. Ván, Remeasurement of the Eötvös-experiment, status and first results, PoS(FFK2019)042, 2019.

[17] E. Fischbach, D. E. Krause, The Eötvös Paradox: The Enduring Significance of Eötvös’ Most Famous Paper, PoS, 2019, https://arxiv.org/abs/1901.11163.

[18] K. Wagoner, et al. Gee Lab's Equivalence Principle Experiment, PoS(FFK2019)043, 2019.

[19] T.A. Wagner, S. Schlamminger, J.H. Gundlach, and E.G. Adelberger, Torsion-balance tests of the weak equivalence principle", Class. Quantum Grav. 29, 184002, 2012.

[20] R. Cowsik, N. Krishnan, P. Saraswat, S.N. Tandon, S. Unnikrishnan, The Fifth Force Experiment at the TIFR, 1988. 